\title{
Fast Detection of Copy-Move Forgery Image using Two Step Search Algorithm
}

\author{
Yong-Dal Shin \\ Dept. of IT \& Securities, Asan Campus, Youngdong University \\ 52-70 Yeunamsan-ro Eumbong-myeon Asan city ChoongNam, Korea \\ ydshin@yd.ac.kr
}

\begin{abstract}
In this paper, we proposed a new fast detection method of copy-move forgery image using two step search algorithm in the spatial domain. We proposed a new two step search algorithm for copy-moved forgery image detection. The performance of the proposed method is experimented on several forged images. Our two step search algorithm reduced $96.82 \%$ computational complexity more than conventional algorithms for copy-move forgery image detection. The block distortion measure (BDM) of the two step search algorithm is sufficient by 2 pixels checking points instead of 64 pixels checking points for exhaustive search in the not copy-moved forgery image regions. Most blocks of the images constitute not copy-moved forgery images. Therefore, we can reduce computational complexity more than conventional methods. We didn't use any exhaustive search method and frequency domain (ex. DCT, Wavelet Transform) to reduce computational complexity in this paper.
\end{abstract}

Keywords: two step search algorithm, copy-move forgery image, fast detection

\section{Introduction}

The availability of advanced high resolution digital cameras, advanced digital image processing technology and photo editing software packages, such as Photoshop, Paintshop Pro, make it relatively easy to create digital forgery from one or multiple images [1]-[15].

Recently, various techniques for tamper or forgery image detection or even recovery have been proposed in the literature. Some techniques have been proposed for image tamper detection. Various watermark techniques have been proposed in recently years, which can be used not only for authentication, but also for being an evidence for the tamper detection.

Despite the fact that the need for detection of digital forgeries has been recognized by the research community, very few publications are currently available. Digital watermarks have been proposed as a means for fragile authentication, content authentication, detection of tampering, localization of changes, and recovery of original content [2]. While digital watermarks can provide useful information about the image integrity and its processing history, the watermark must be present in the image before the tampering occurs [1]. This limits their application to controlled environments that include military systems or surveillance cameras. Unless all digital acquisition devices are equipped with a watermarking chip, it will be unlikely that a forgery-in the- wild will be detectable using a watermark [1].

Watermark techniques embedded watermarks consisting of the authentication data and the recovery data into image blocks for image tamper detection and recovery in the future [1]-[6]. 
A common form of digital tampering is copy-move forgery, in which a part of the image itself is copied and pasted into another part of the same image to conceal an important object [7]. The goal in detection of copy-move forgeries is to detect image areas that are same or extremely similar [8]. Because the copied part come from the same image, its important properties, such as noise, color and texture, will be compatible with the rest of the image and thus will be more difficult to distinguish and detect [7].

An example of a digital forgery is shown in Figure 1. The original image of a figure 1 is shown figure 1(a). The figure 1(a) is shown 3 cars on the land. The forgery image of the figure 1 is shown figure 1(b). The figure 1(b) is shown 4 cars on the land. A car of the figure 1(b) is forgery image block. Therefore, the figure 1 is example of the copy-moved forgery image. Forgery image detection is to detect the counterfeit image.

Copy move forgery is one type of tempering that is commonly used for manipulating the digital contents; in this case, a part of an image is copied and is pasted on another region of the image [9]. The focus of this paper is on detecting copy-move forgery, because most of the tampering involves hiding or adding some image contents and it is accomplished by copy and move. The task of tamper detection becomes more difficult with the copy move forgery [9]. This is due to the similarity between the characteristics of the copied and pasted regions of an image; these characteristics include the noise component, color palette, dynamic range etc [9]. This indicates that the detection methods that search for tampered image regions using inconsistencies in statistical measures will fail [9].

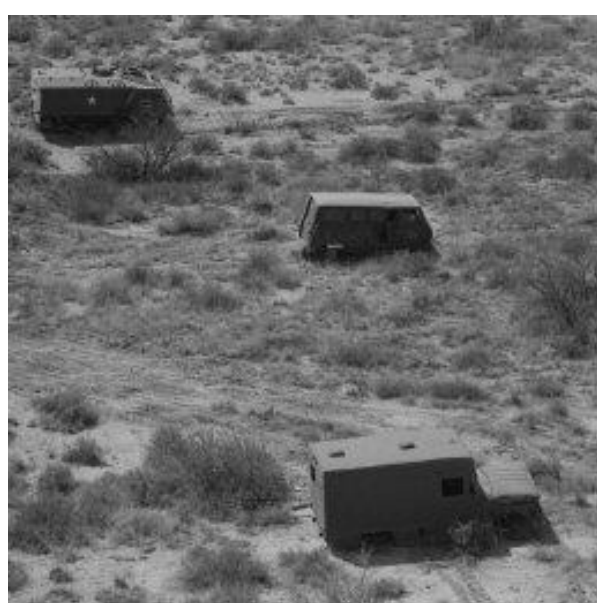

(a)

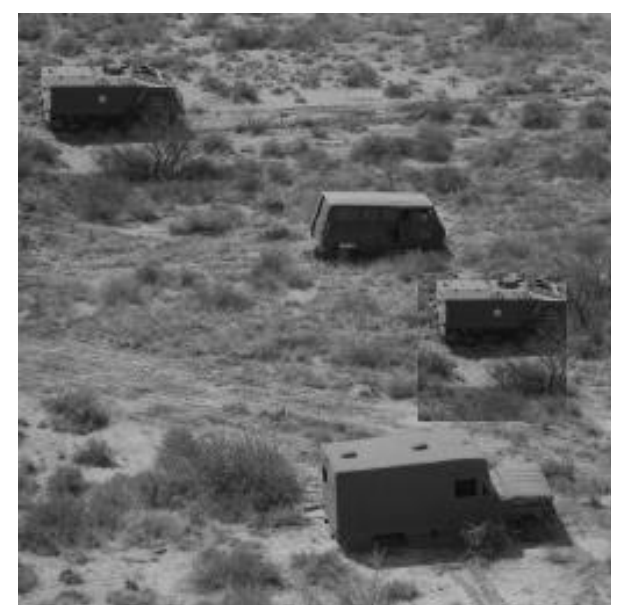

(b) Figure 1. Example of Copy-Move forgery (a) Original Image (b)
Tampered Image

J. Fridrich [1] proposed exact match for detection and an effective blocking approach, in which the image blocks are represented by quantized discrete cosine transform coefficients, and a lexicographic sort is adopted to detect the Copy-Move blocks [1]. A. C. Popescu [2] worked by applying a principal component analysis (PCA) on small fixed size image blocks to yield a reduced dimension representation.

In most methods of copy-move forgery detection, the detected image is divided into overlapping blocks. Suppose a detected image of size $\mathrm{N} \times \mathrm{N}$ is divided into ( $\mathrm{N}$ $\mathrm{B}+1)^{2}$ overlapping blocks of size $\mathrm{B} \times \mathrm{B}[6]$. Copy-move forgery image detection methods is demanded huge computation of the exhaustive search method in the 
spatial domain [1], also demanded computational complexity in the frequency domain such as discrete cosine transform (DCT) and wavelet transform [1], [4], [7].

Y. D. Shin [10] worked by applying a three step search algorithm in the spatial domain to reduce computation complexity. The three step search algorithm was introduced by Koga et al [11]. applied motion estimation for moving pictures. Y. D. Shin [12] worked by applying DCT algorithm to reduce computation complexity.

In this paper, we proposed a new fast detection method of copy-move forgery image using two step search algorithm in the spatial domain. We proposed a new two step search algorithm for copy-moved forgery image detection. The performance of the proposed method is experimented on several forged images. Our two step search algorithm reduced $96.82 \%$ computational complexity more than conventional algorithms for copy-move forgery image detection. In this paper, the computation number of the two step search algorithm is 2 pixels or 64 pixels checking points for $8 \times 8$ pixel block. The block distortion measure (BDM) of the two step search algorithm is sufficient by 2 pixels checking points instead of 64 pixels checking points for exhaustive search in the not copy-moved forgery image regions. Most blocks of the images constitute not copy-moved forgery image. Therefore, we can reduce computational complexity more than conventional methods. We didn't use any exhaustive search method and frequency domain (ex. DCT, Wavelet Transform) to reduce computational complexity in this paper.

\section{The Proposed Method}

Copy-move forgery image detection means a correlation between the original image blocks and the pasted image blocks [1]. The correlation can be used as a basis for fully detection of type of a counterfeit image [1]. The conventional copy-move forgery image detection methods are demanded huge computation of exhaustive search method in the spatial domain [1], also demanded computational complexity in the frequency domain such as DCT [14] and wavelet transform [1][4][7]. The exhaustive search algorithm is the simplest and most obvious approach [1]. Even though the simple exhaustive search algorithm is effective, but it is a huge computational complexity. Thus, the exhaustive search algorithm could not applied for medium-size images [1].

In order to detect the forgery image regions, let us suppose that the region is a square block with $B \times B$ pixels and image size is $M \times N$ pixels. The square is slid by one pixel along the image from the upper left corner right and down to the lower right corner. For each position of the $B \times B$ pixel block, the pixel values from the block are extracted by columns into a row of a two-dimensional array $A$ with $B^{2}$ columns and $(M-B+1)(N-B+1)$ rows. Each row corresponds to one position of the sliding block [1].

In this paper, we proposed fast detection method of copy-move forgery image using two step search algorithm in the spatial domain. We didn't use any exhaustive search method and frequency domain to reduce computation complexity.

The proposed two step search algorithm described as:

Step 1: The first step explore shading 2 pixels checking points of the $8 \times 8$ pixel block in the figure 2 . The first step required 2 pixels checking points to explore pre-copy-moved forgery image block. The shading 2 pixels of the first step were computed by equation (3), (4), and (5).

Step 2: The second step explore 62 pixels checking points except 2 pixels shading of the $8 \times 8$ pixel block in the figure 2 . The second step yields the horizontal $\mathrm{x}$ and vertical $\mathrm{y}$ motion vector of the copy-moved forgery image. The motion vector $\mathrm{x}$ and $\mathrm{y}$ are the location of the copy-moved image block.

The shading 2 pixels of the first step and 62 pixels of the second step were computed by equation (3), (4), and (5) to find motion vector $\mathrm{x}$ and $\mathrm{y}$. 
In our algorithm, we need 2 pixels for not copy-moved image blocks of the forgery image and 64 pixels for copy-moved image blocks of the forgery image. We only computed 2 pixels for not copy-move forgery images. There are many blocks not copymoved image block in the images. So the proposed algorithm can reduce compute complexity more than conventional methods.

\begin{tabular}{|c|c|c|c|c|c|c|c|}
\hline 1 & 2 & 3 & 4 & 5 & 6 & 7 & 8 \\
\hline 9 & 10 & 11 & 12 & 13 & 14 & 15 & 16 \\
\hline 17 & 18 & 19 & 20 & 21 & 22 & 23 & 24 \\
\hline 25 & 26 & 27 & 28 & 29 & 30 & 31 & 32 \\
\hline 33 & 34 & 35 & 36 & 37 & 38 & 39 & 40 \\
\hline 41 & 42 & 43 & 44 & 45 & 46 & 47 & 48 \\
\hline 49 & 50 & 51 & 52 & 53 & 54 & 55 & 56 \\
\hline 57 & 58 & 59 & 60 & 61 & 62 & 63 & 64 \\
\hline
\end{tabular}

Figure 2. 8×8 Pixel Block for Two Step Search Algorithm

We used block distortion measure (BDM) for matching criterion (MC) of copy-moved forgery image detection. The BDM expressed equation (3).

$$
\mathrm{BDM}=\Sigma|\mathrm{I}(\mathrm{i}, \mathrm{j})-\mathrm{I}(\mathrm{i}+\mathrm{x}+\mathrm{ii}, \mathrm{j}+\mathrm{y}+\mathrm{jj})|
$$

where, $I(i, j)$ is gray level of $8 \times 8$ pixel block at the position $(i, j)$ of criteria image region, the $i$ and $j=0,1,2,3 \ldots, B-1$. Where the block size $B$ is 8 . The position $i$ and $j$ are shifted one pixel vertical and horizontal of the image from 0 to $\mathrm{N}-\mathrm{B}+1$. In the matching search area, $\mathrm{ii}=0,1$ and $\mathrm{jj}=0$ for the first step, $\mathrm{ii}=2 \sim \mathrm{B}-1, \mathrm{jj}=1 \sim \mathrm{B}-1$ for the second step. The position $\mathrm{x}$ and $\mathrm{y}$ are shifted one pixel vertical and horizontal of the image from 0 to $\mathrm{N}-\mathrm{B}+1$ overlapping blocks in the copy-moved forgery matching search regions.

The BDM is summation of difference between gray level of criteria image block and copy-move forgery image matching search regions. We used a matching criterion (MC) of copy-moved forgery image detection. The MC is expressed by eq. (4) and (5).

1) The first step for 2 pixels (using 2 pixels of figure 2)

If $(\mathrm{BDM}==0)$

Else

$$
\text { Pre-Copy-moved forgery block }
$$

Not copy-moved forgery block

2) The second step for 62 pixels (using 62 pixels of figure 2)

$$
\begin{aligned}
& \text { If }(\text { BDM }==0) \\
& \text { Copy-moved forgery block }
\end{aligned}
$$

Else

Not copy-moved forgery block 
From equation (4), if the BDM were 0 , the block is a pre-copy-moved forgery image block. From equation (5), if the BDM were 0, the block is a copy-moved forgery image block. This means that the copied block image is same moved block image. If the BDM were not 0 , the block is not copy-move forgery image block. This means that the copied image block is different moved image block. So, we do not computer 62 pixels of the block. The flow chart of proposed method is shown by figure 3 .

The proposed method reduced computational complexity because it doesn't frequency domain such as discrete cosine transform and wavelet transform. In the copy-moved forgery image blocks require 64 (step1,2) computation complexity, but not forgery image blocks need 2 (step 1) computation complexity to match duplicated pairs images. So, we can reduce computational complexity.

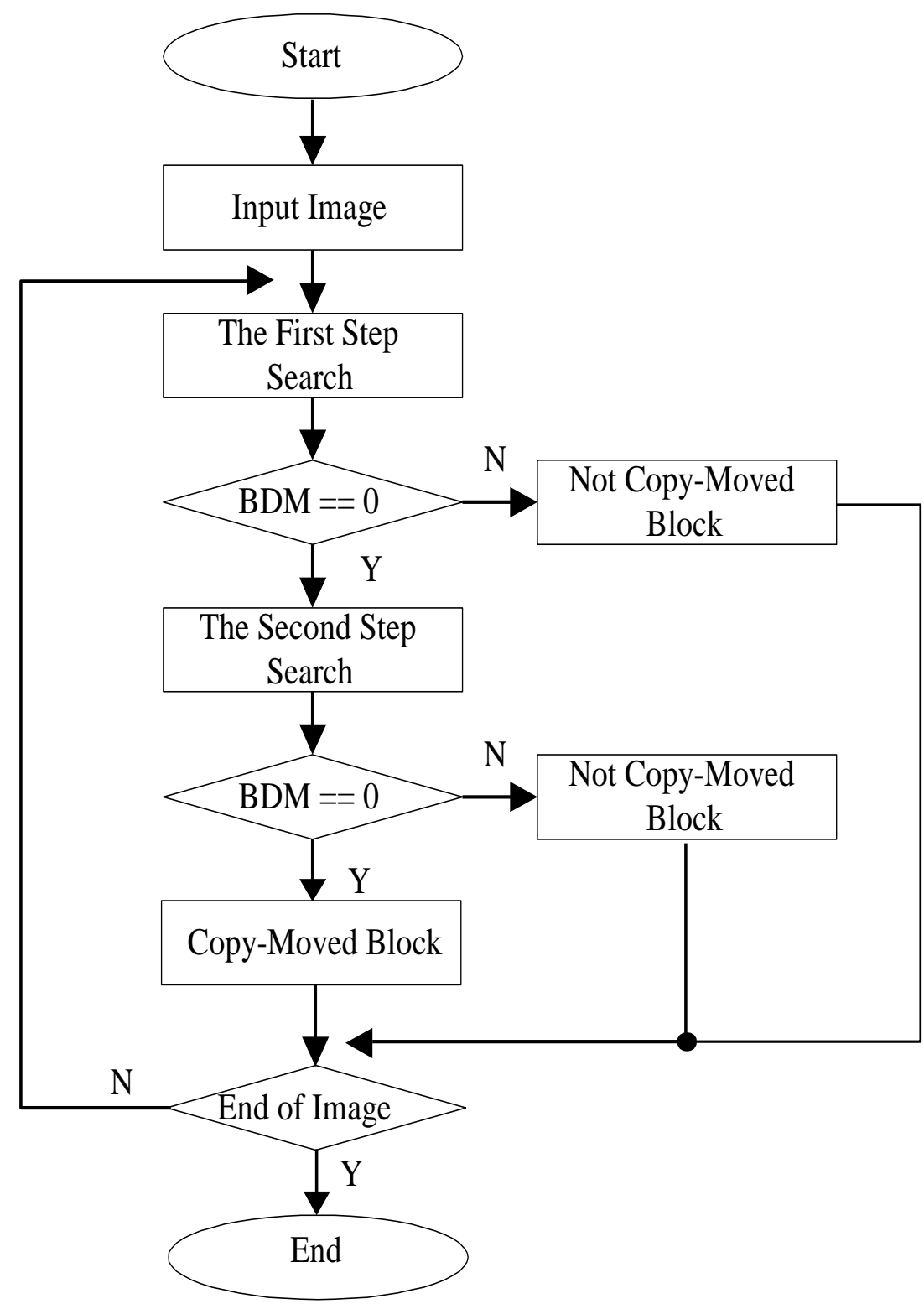

Figure 3. The Flow Chart of Proposed Method using Two Step Search Algorithm 


\section{Experimental Results}

We proposed a new fast method to explore copy moved forgery image using two step search algorithm in the spatial domain. The proposed method reduced computational complexity more than conventional methods.

The test images are Bridge, Tcar3, Lake, House images, 8 bits grey level and $256 \times 256$ pixels. We tampered test images by copy-moved forgery image. The copymoved forgery search regions of test images are shifted by one pixel from the upper left corner $\mathrm{I}(0,0)$ to the lower right corner $\mathrm{I}\left(\mathrm{N}-\mathrm{B}+1, \mathrm{~N} \_\mathrm{B}+1\right), \mathrm{N}=256$, overlapping blocks. The BDM of copy-moved forgery image detection used equation (3), (4) and (5) based on two step search algorithm. From equation (3), (4) and (5), if the BDM were 0 , the block is a copy-moved forgery block. This means that the copied block image is same moved block image. If the BDM were not 0 , the block is not copymove forgery block. This means that the copied block image is different moved block image.

In this paper, the computation number of the two step search algorithm is 2 pixels or 64 pixels checking points for $8 \times 8$ pixel block. The BDM of the two step search algorithm is sufficient by 2 pixels checking points instead of 64 pixels checking points (exhaustive search) for not copy-moved forgery image. The most of the images constitute not copy-moved forgery image. Therefore, we can reduce computational complexity more than conventional methods.

The table 1 showed computational complexity results of the proposed method and conventional methods for test 4 images (Bridge, Tcar3, Lake, House), such as figure $4,5,6$, and 7 . The reference value for computation complexity used $100 \%$ by references [1] and exhaustive search algorithm.

The table 2 showed results of the proposed method for 4 test images and Average computation complexity of 4 test images (Bridge, Tcar3, Lake, House), such as figure $4,5,6$, and 7 . The proposed method perfectly $100 \%$ detected every copymove forgery image blocks.

From table 1, algorithms, image representation, copy-moved matching search region block number, feature dimension, computational complexity (based on reference [1]). The computational complexity of various conventional methods compared exhaustive search, Fridrich[1], Popescu[2], Kahn[7], and Shin [10].

From table 2, the computational complexity is different from test images. Because the equation (3), (4), and (5) of the proposed two search algorithm can be different from test images. Thus we used average computational complexity for 4 test images.

From table 2, the average computational complexity for 4 test images of proposed method was $3.18 \%$, when the reference [1] as $100 \%$.

Table 1. Comparison Results of Conventional Method for Test Images

\begin{tabular}{|c|c|c|c|c|}
\hline Algorithms & $\begin{array}{c}\text { Image } \\
\text { representation }\end{array}$ & $\begin{array}{c}\text { Block } \\
\text { number }\end{array}$ & $\begin{array}{c}\text { Feature } \\
\text { dimension }\end{array}$ & $\begin{array}{c}\text { Computation } \\
\text { complexity } \\
\text { ( Ref. [1]) }\end{array}$ \\
\hline Exhaustive & Gray level & $(256-8+1)^{2}$ & 64 & $100.0 \%$ \\
\hline Fridrich[1] & DCT & $(256-8+1)^{2}$ & 64 & $100.0 \%$ \\
\hline Popescu[2] & PCA & $(256-8+1)^{2}$ & 32 & $50.00 \%$ \\
\hline Kahn[7] & DWT & $(128-8+1)^{2}$ & 64 & $26.01 \%$ \\
\hline
\end{tabular}




\begin{tabular}{|c|c|c|c|c|}
\hline Shin[10] & $\begin{array}{c}\text { Gray level } \\
\text { (TSS) }\end{array}$ & $25 \times 17^{2}$ & 64 & $11.65 \%$ \\
\hline $\begin{array}{c}\text { Proposed } \\
\text { method }\end{array}$ & $\begin{array}{c}\text { Gray level } \\
\text { (Average of } 4 \\
\text { test images) }\end{array}$ & $(256-8+1)^{2}$ & 2 or 64 & $3.18 \%$ \\
\hline
\end{tabular}

Table 2. Results of the Proposed Method for Test Images

\begin{tabular}{|c|c|c|c|c|}
\hline Test images & $\begin{array}{c}\text { Block } \\
\text { size }\end{array}$ & $\begin{array}{c}\text { Block } \\
\text { number }\end{array}$ & $\begin{array}{c}\text { Feature } \\
\text { dimension }\end{array}$ & $\begin{array}{c}\text { Computation } \\
\text { complexity } \\
\text { ( Ref. [1]) }\end{array}$ \\
\hline Bridge & $8 \times 8$ & $(256-8+1)^{2}$ & 2 or 64 & $3.13 \%$ \\
\hline Tcar3 & $8 \times 8$ & $(256-8+1)^{2}$ & 2 or 64 & $3.16 \%$ \\
\hline Lake & $8 \times 8$ & $(256-8+1)^{2}$ & 2 or 64 & $3.17 \%$ \\
\hline House & $8 \times 8$ & $(256-8+1)^{2}$ & 2 or 64 & $3.25 \%$ \\
\hline \multicolumn{2}{|r|}{ Average computation complexity of 4 test images } & $3.18 \%$ \\
\hline
\end{tabular}

The proposed method reduced $96.82 \%$ of computational complexity than exhaustive search algorithm [1]. From table 1, the proposed method reduced computational complexity more than conventional method [1][2][7]. The table 2 is shown by average computation complexity of 4 test images. Average computation complexity of 4 test images is $3.18 \%$.

The figure 4, 5, 6 and 7 showed results of proposed method. We showed (a) original images, (b) copy-move forgery images, (c) detection of copy-move forgery images in the figure $4,5,6$, and 7 respectively.

From Bridge image of the figure 4 (c) and Tcar3 image figure 5(c), the left black box is copied image block, the right black box is moved forgery image block.

From Lake image of the figure 6 (c) and House image of the figure 7(c), right black box is copied image block and left black box is moved forgery image block.

From figure 4(c), 5(c), 6(c) and 7(c), our algorithm detected copy-move forgery images perfectly and largely reduced computational complexity more than conventional methods. 


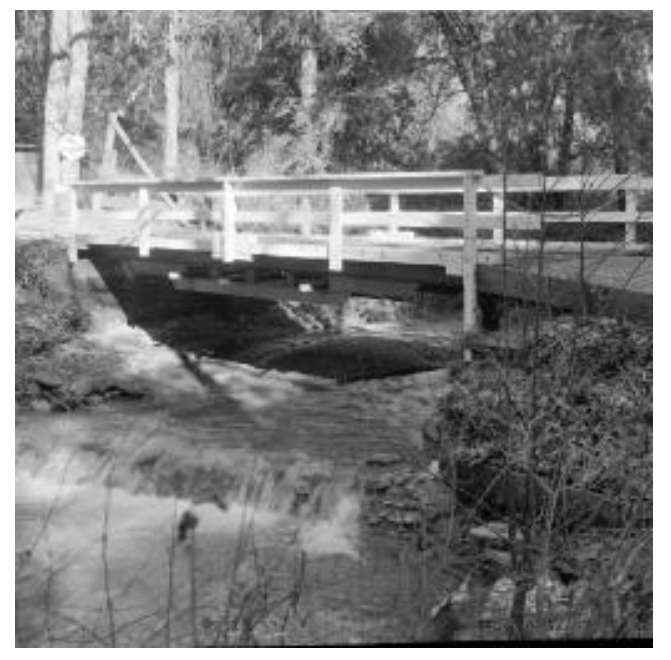

(a) Original Image

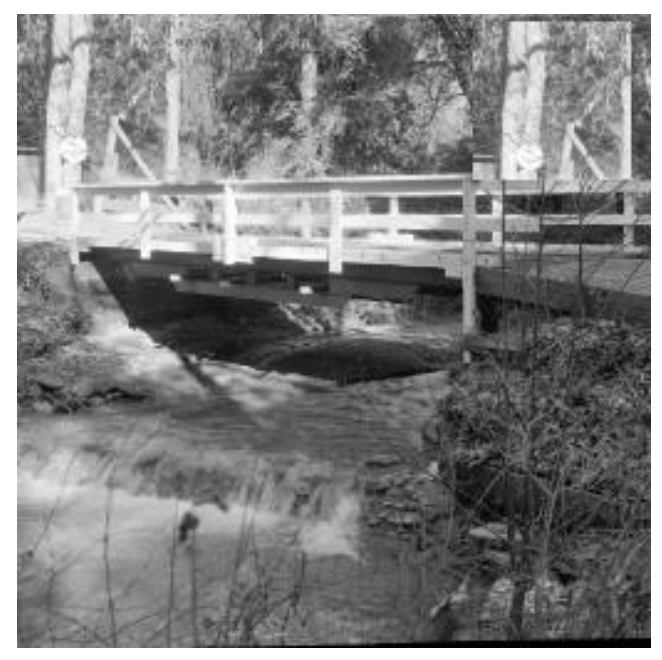

(b) Copy-move forgery

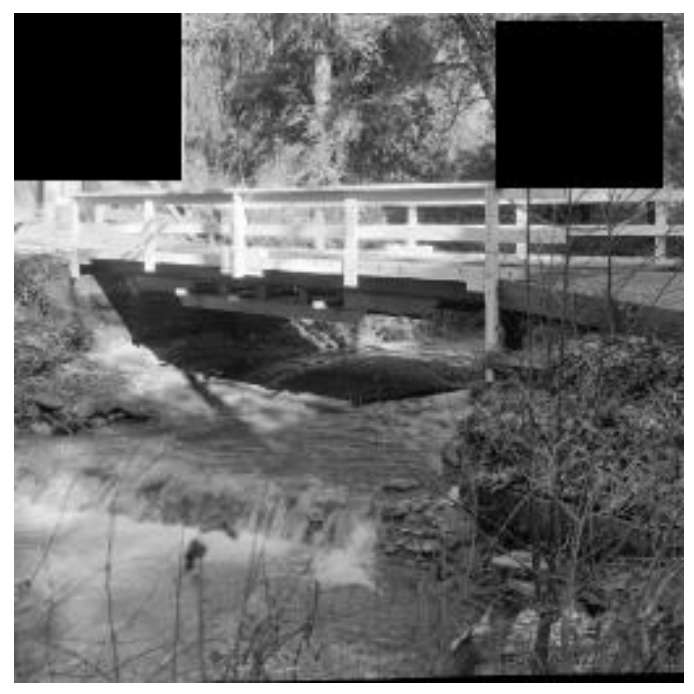

(c) Detection of copy-move forgery (Black box)

Figure 4. Bridge Image 


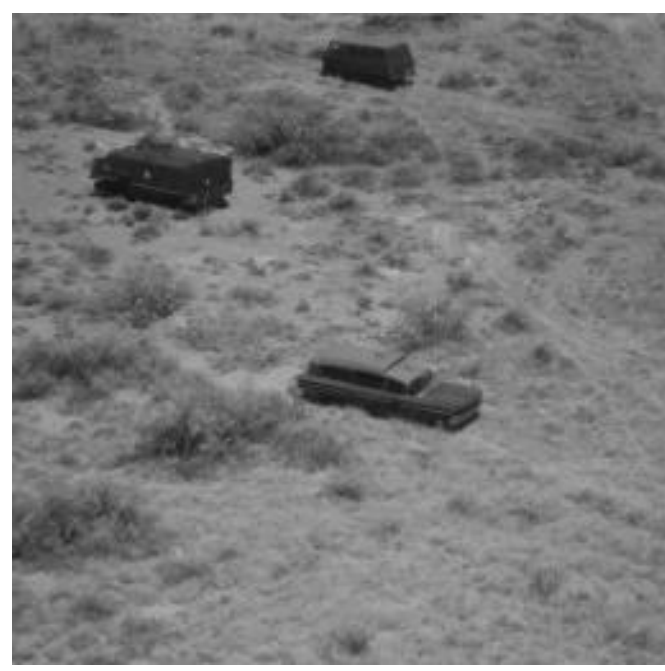

(a) Original image

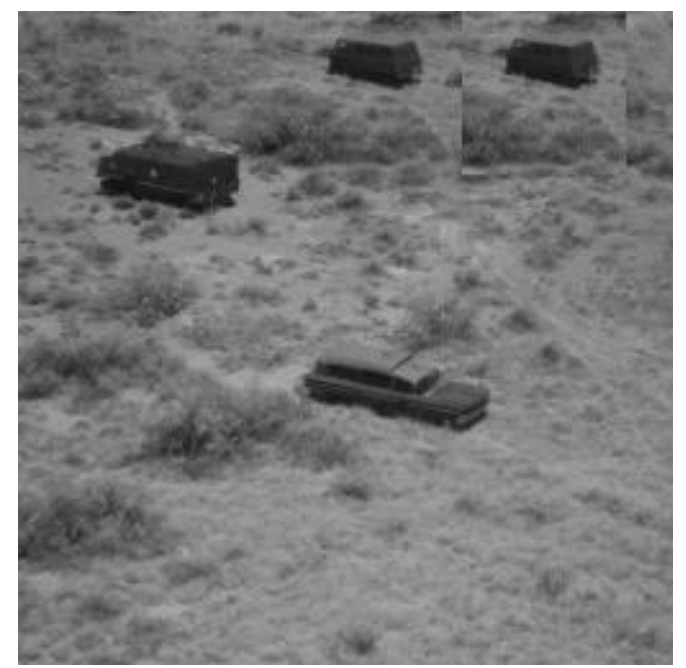

(b) Copy-moved forgery image

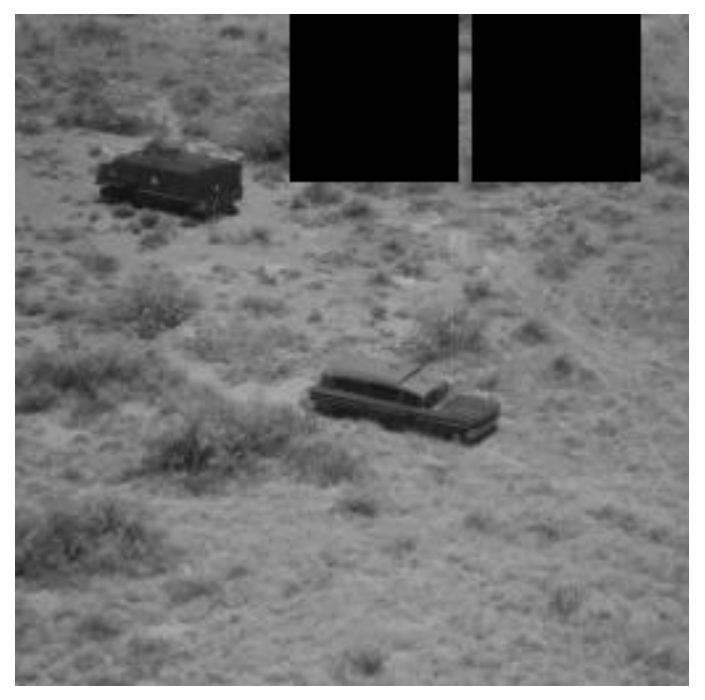

(c) Detection of copy-moved forgery image (Black box)

Figure 5. Tcar3 Image 


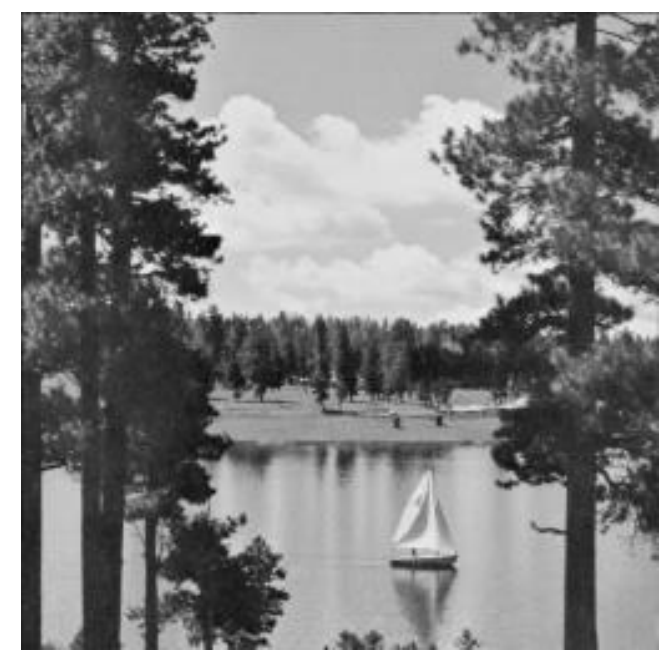

(a) Original Image

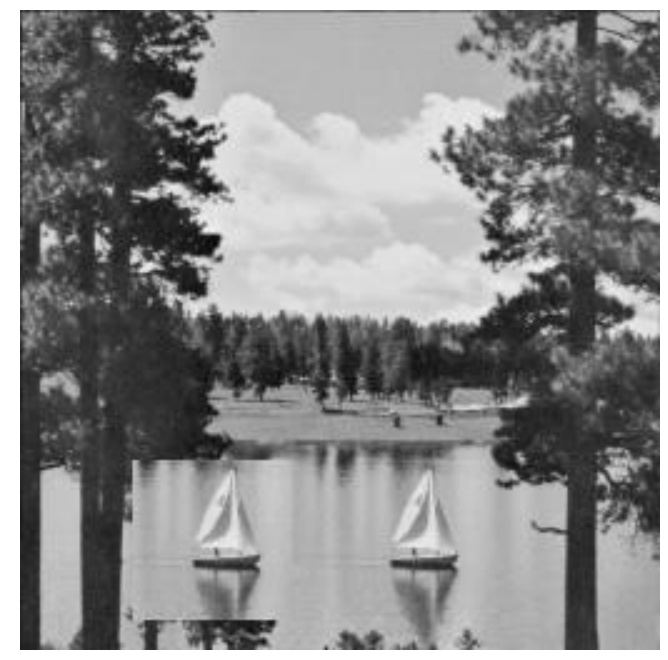

(b) Copy-move forgery

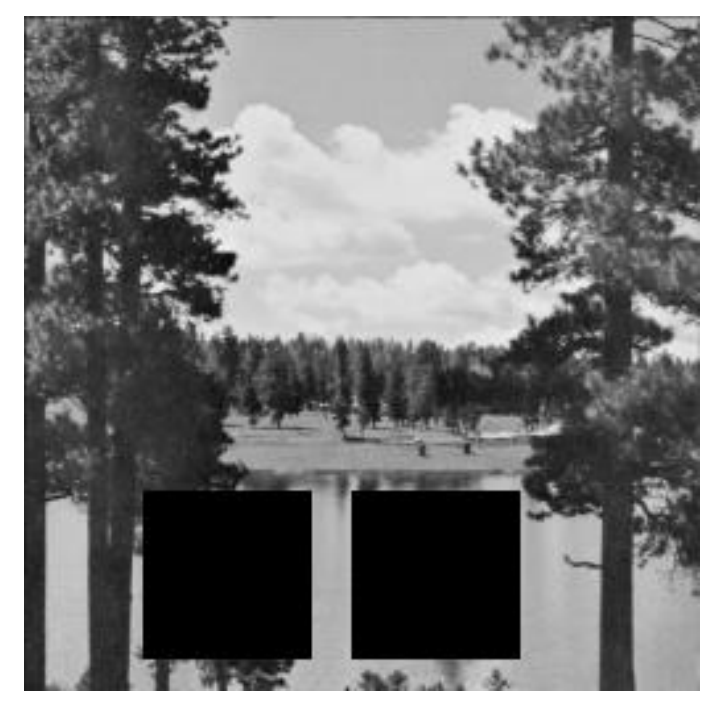

(c) Detection of copy-move forgery (Black box)

Figure 6. Lake Image 


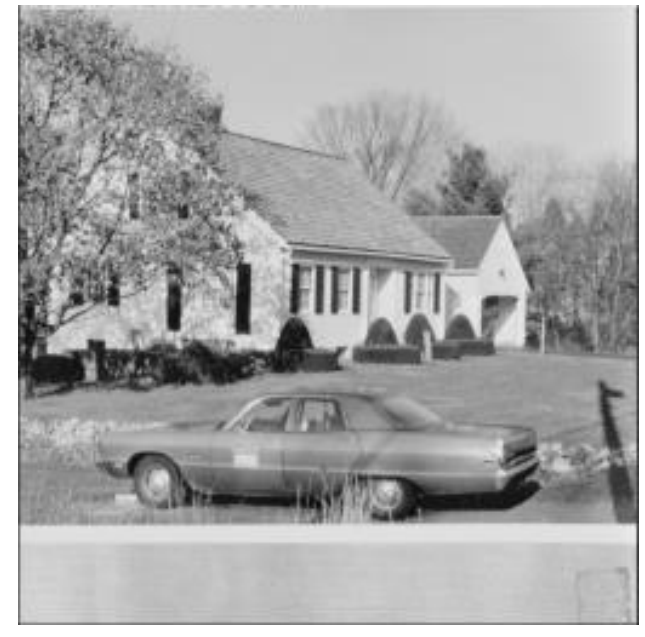

(a) Original Image

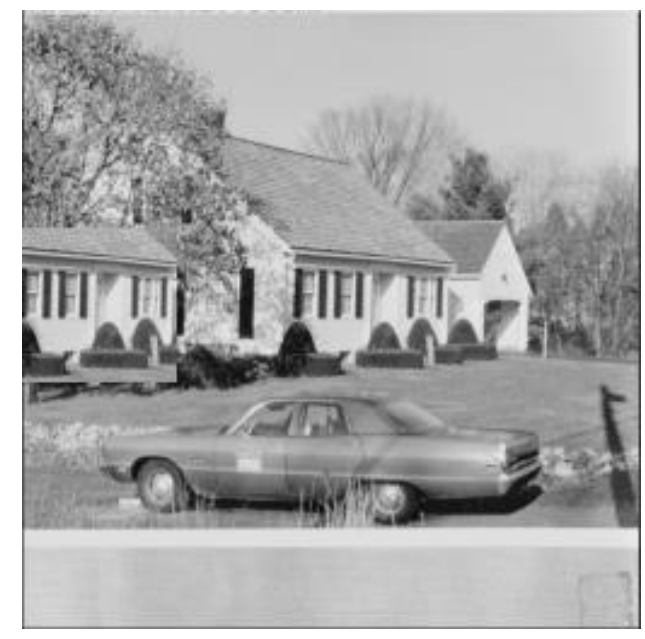

(b) Copy-move forgery

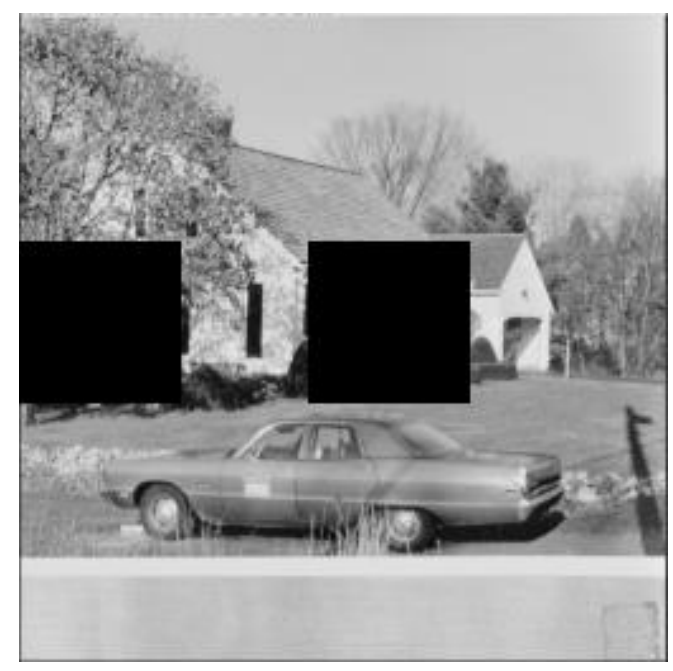

(c)Detection of copy-move forgery (Black box)

Figure 7. House image

\section{Conclusions}

In this paper, we proposed a new fast detection method of copy-move forgery image using two step search algorithm in the spatial domain. We proposed a new two step search algorithm for copy-moved forgery image detection. The performance of the proposed method is experimented on several forged images. Our two step search algorithm reduced $96.82 \%$ computational complexity more than conventional algorithms for copy-move forgery image detection. In this paper, the computation number of the two step search algorithm is 2 pixels or 64 pixels 
checking points for $8 \times 8$ pixel block. The block distortion measure (BDM) of the two step search algorithm is sufficient by 2 pixels checking points instead of 64 pixels checking points for exhaustive search in the not copy-moved forgery image regions. Most blocks of the images constitute not copy-moved forgery image. Therefore, we can reduce computational complexity more than conventional methods. We didn't use any exhaustive search method and frequency domain (ex. DCT, Wavelet Transform) to reduce computational complexity in this paper.

\section{References}

[1] J. Fridrich, D. Soukal, J. Lukas "Detection of copy-move forgery in digital images. Proceedings of Digital Forensic ", Research Workshop (2003).

[2] J. Fridrich, "Methods for "Methods for Tamper Detection in Digital Images", Proceedings ACM Workshop on Multimedia and Security, Orlando, FL, October 30-31, (1999), pp. 19-23.

[3] A. C. Popescu, H. Farid, "Exposing digital forgeries by detecting duplicated image regions", Dartmouth College, Hanover, New Hampshire, USA:TR2004-515, ( 2004).

[4] J. Zhang, Z. Feng, Y. Su, "A new approach for detecting copy-move forgery in digital images", $11^{\text {th }}$ IEEE Singapore International Conference on Communication Systems (2008) pp. 362-366.

[5] B. G. Jeong, I. K. Eom, “ Detection of copy-move forgery image wavelet domain”, Proceeding of the 23-th Korean Signal Processing Conference, vol.23 , (2010).

[6] E. S. Khan, E. A. Kulkarni, “ An efficient method for detection of copy-move forgery using discrete wavelet transform”, International Journal of Computer Science and Engineering vol. 2, no. 5, (2010), pp. 1801-1806.

[7] H. J. Lin, C. W. Wang, Y. T. Kao, "Fast copy-move forgery detection", WSEAS Transaction on Signal Processing vol. 5, issue 5, (2009), pp. 188-197.

[8] S. Kahn, A. Kulkarni, "Reduced time complexity for detection of copy-move forgery using discrete wavelet transform", International Journal of Computer Applications vol. 6 no.7, (2010), pp. 31-36.

[9] N. Muhammad1, M. Hussain, G. Muhammad, and G. Bebis, "Copy-move forgery detection using dyadic wavelet transform", Proceedings of IEEE Eighth International Conference on Computer Graphics, Imaging and Visualization (CGIV), (2011), pp. 103-108.

[10] S. Bayram, H. T. Sencar, and N. memon, "A survey of copy-move forgery detection techniques", IEEE Western New York Image Processing Workshop (2008).

[11] Y. D. Shin, "Fast detection of copy-move forgery image using three step search algorithm in the spatial domain", ICHIT Lecture Notes in Computer science vol.7425, (2012), pp.389-395.

[12] T. Koga , K. Iinuma, A. Hirano, Y. Iijima and T. Ishiguro, "Motion compensated interframe coding for video conferencing", Procedings. NTC 81, (1981), pp. C9.6.1-9.6.5.

[13] Y. D. Shin, Fast Detection of copy-move forgery image using DCT”, Journal of Korea Multimedia Society vol. 16, no.4, (2013), pp.411-4171.

[14] S. Bayram, H. T. Sencar, and N. memon, "An efficient and robust method for detecting copy-move forgery”, IEEE ICASSP, Taipei, (2009), pp.1053-1056.

[15] Y. D. Shin, "Fast exploration of copy-move forgery image", Proceedings of the 10th International Conference on Information Security and Assurance, Jeju, Korea (2016).

[16] P. Yip, K. Rao, Discrete cosine transform: algorithms, and applications, Academic press, (1990)

[17] http://sipi.usc.edu/database.

[18] http://www.ece.cmu.edu/ ee899/project/deepak_mid.htm.

[19] http://sipl.kjist.ac.kr/about/2-Famous\%20ME\%20algorithms.pdf.

\section{Authors}

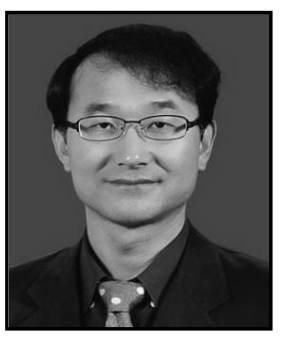

Yong-Dal Shin, he received his B.E., M.S., and $\mathrm{PhD}$ degree in electronics from Kyungpook national university, Korea. He is currently a professor department of the IT \& securities at the Asan campus, Youngdong university, Korea. His current research interest includes image security, steganography, digital watermark and digital forensics. 